\title{
The application of parallel robotics to investigate the effect of lumbar bracing on trunk muscle activity
}

\author{
Gregory N. Kawchuk ${ }^{\mathrm{a} *}$, Narasimha G. Prasad ${ }^{\mathrm{b}}$, Jonathan Glass ${ }^{\mathrm{a}}$, Carolyn Knight ${ }^{\mathrm{a}}$, Serena Third ${ }^{\mathrm{a}}$ and Daniel Timmermans ${ }^{\mathrm{a}}$ \\ ${ }^{a}$ Department of Physical Therapy, University of Alberta, Edmonton, Canada; ${ }^{b}$ Department of Math and Statistical Sciences, University \\ of Alberta, Edmonton, Canada
}

(Received 28 January 2010; final version received 28 July 2010)

\begin{abstract}
Lumbar bracing is prescribed frequently for disability caused by low back pain; however, investigations into this practice demonstrate a range of patient outcomes. This inconsistency may arise from the practice of employing voluntary, single-axis trunk movements when investigating braces. Alternatively, this study employed a parallel robot to create a standardised, multi-axis testing environment. Surface electromyographic (sEMG) data were collected from the trunk of 24 asymptomatic participants, who were seated on the robot, tilted to $15^{\circ}$, then circumducted while attempting to maintain an upright posture. Multiple trials were performed for three randomised conditions: non-braced, soft-material brace and stiff-material brace. As expected, the sEMG activity was significantly reduced in the majority of muscle responses (201/240). Unexpectedly, a paradoxical increase in the sEMG activity was observed in 39/240 responses. While lumbar bracing reduces the sEMG activity on average, these data suggest the existence of an infrequent paradoxical response that may provide a possible explanation for the discordant results observed in previous bracing investigations.
\end{abstract}

Keywords: trunk; electromyography; bracing; muscle; robotics

\section{Introduction}

Lumbar orthoses (i.e. braces) are thought to improve posture and body mechanics, reduce muscle fatigue and decrease compressive loading on the spine (Poppel et al. 2000). As a result, lumbar orthoses are often prescribed for disability caused by low back pain (LBP) or as a preventative measure for people with physically demanding occupations. Despite their common use for these conditions, the mechanism by which lumbar braces exert their effect to prevent, or treat low back pain, is not clearly understood.

Several hypotheses exist regarding the possible mechanisms underlying lumbar bracing that include restriction of extreme ranges of motion, thereby protecting the spine from injury (Buchalter et al. 1988; Cholewicki et al. 2003), increasing intra-abdominal pressure to increase spine stability (McGill et al. 1994) and improved proprioception as a tactile reminder to avoid potentially dangerous postures (McNair and Heine 1999; Poppel et al. 2000).

Unfortunately, the results from studies that have investigated the effect of lumbar bracing on trunk muscle activities are inconsistent. In a systematic review, van Poppel et al. (2000) reported on 11 studies, three of which demonstrated inconclusive evidence, four demonstrated decreased muscle activity while braced and remaining four studies described no difference between the braced and un-braced conditions. In addition, other investigators
(Ivancic et al. 2002) have found that activity in spinal and abdominal musculature did not change between braced and non-braced conditions despite a reported increase in passive spinal stiffness. Similarly, McGill et al. (1990) did not observe a change in muscle activity during squatting activities while subjects were braced.

One explanation for the variation in the outcomes of these studies is a lack of consistency in subject-generated movements. Generally, movements employed in these previous studies are uni-axial, voluntary and uncontrolled. Standardising subject activity may provide increased consistency in outcome measures related to brace performance (Anders et al. 2008).

To explore the relation between muscle activity and a standardised kinematic environment, Kawchuk et al. (2008) have previously employed a parallel robot. Specifically, subjects in this study were seated on a parallel robot, tilted to an ascension angle between $5^{\circ}$ and $15^{\circ}$, then circumducted $360^{\circ}$. During circumduction, subjects were asked to maintain an upright posture by training a laser strapped to their chest on a distant target. Maintaining an upright posture through $360^{\circ}$ of tilted circumduction resulted in the activation of trunk muscles, as determined by surface electromyography (sEMG). With this approach, the authors found that spinal muscle activity was correlated positively with the tilt angle of the robotic platform (Kawchuk et al. 2008). It was concluded that this methodology provided the 
Table 1. Inclusion and exclusion criteria for subject recruitment.

Inclusion criteria

- Males/females between the ages of 18 years and 65 years.

Exclusion criteria

- History of low back pain within the last three months prior to testing.

- Previous back surgery or any lower extremity injury with continuing symptoms or complications.

- Balance complaints or episodes of dizziness.

- Skin conditions or neurological deficits, which interfere with the use of sEMG electrodes.

- Pregnancy or suspicion of pregnancy.

- Medications that could affect muscle function or the ability to identify painful stimuli.

means to capture effective trunk sEMG within a standardised protocol.

To date, we are aware of no study that has combined the use of a standardised, multi-axial movement with sEMG to assess lumbar bracing. As a result, the purpose of this study was to measure trunk muscle activity in asymptomatic subjects during standardised multi-axial trunk movement for three conditions - no brace, soft-material lumbar brace and stiff-material lumbar brace. Given this, our hypothesis was that lumbar bracing reduces trunk muscle activity. It is expected that findings from this study will increase our understanding regarding the underlying mechanisms by which bracing may help those with low back pain.

\section{Methods}

\subsection{Overview}

The surface electromyography data from five paired sites were collected simultaneously as subjects were seated on a robotic platform that performed combined tilt and circumduction. Participants completed three trials for each of three test conditions: no brace, soft-material brace, and stiff-material brace. Following non-braced trials, testing order was randomised between the soft-material and stiffmaterial brace conditions.

\subsection{Participants}

Ethics approval for the study was received from the University of Alberta Health Research Board. Twenty-four asymptomatic participants were recruited to the study, each of whom met the study's inclusion criteria (Table 1).

\subsection{Lumbar braces}

Lumbar braces were provided by Aspen Medical Products Inc. (CA, USA). The QuikDraw ${ }^{\mathrm{TM}}$ brace was used for the soft-material brace (Figure 1). This brace, made of deformable elastic, is presumed to maintain a constant intrabrace volume during subject movement through a series of transverse cords used to tighten the brace. For the stiffmaterial brace, the lumbar sacral orthosis (LSO) product (Lumbosacral Bracing System) was employed (Figure 1).
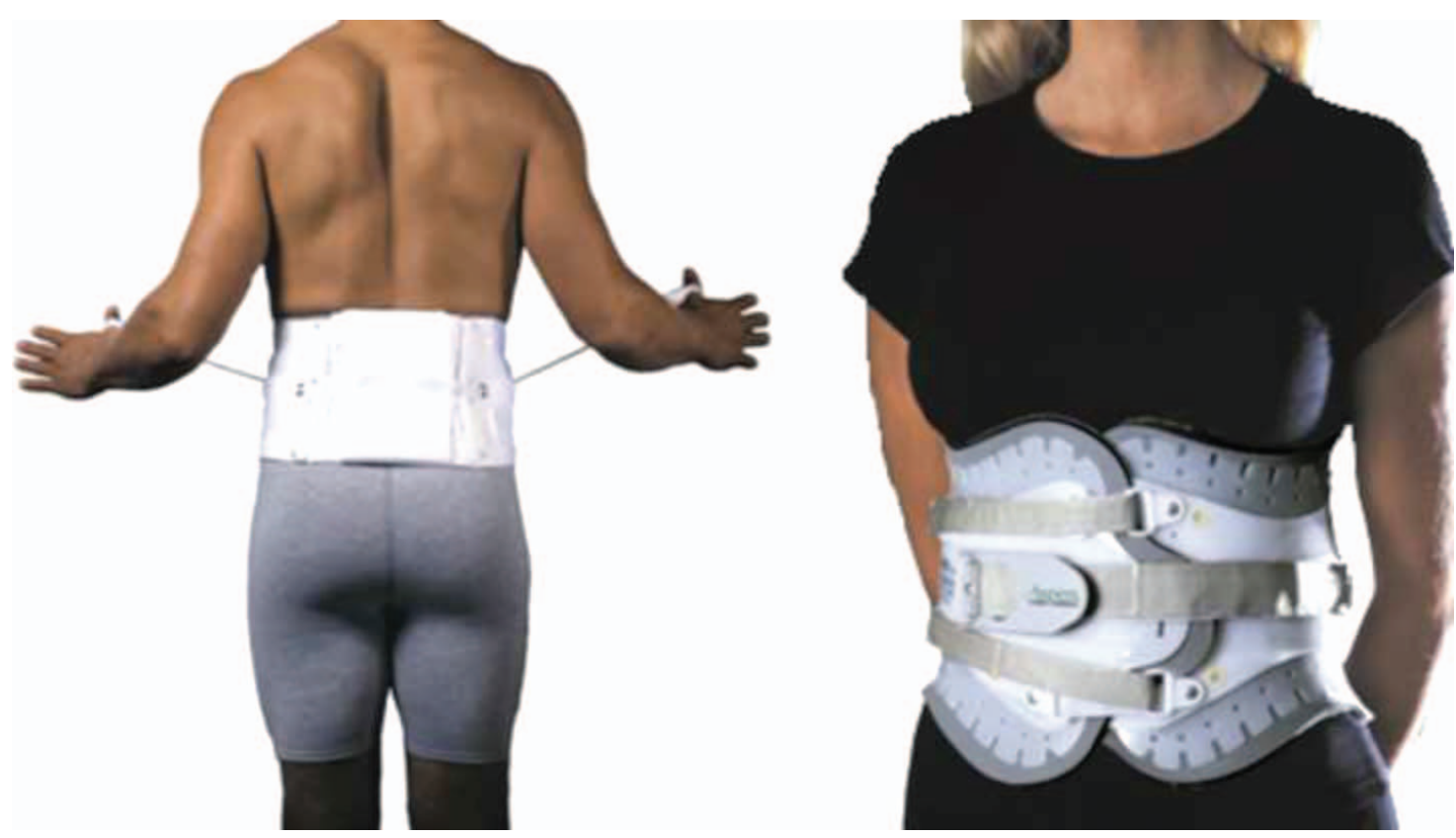

Figure 1. The two braces used in this experiment. The soft-material brace (left) was the QuikDraw ${ }^{\mathrm{TM}}$ RAP (Aspen Medical Products Inc., Irvine, CA), which could be tightened circumferentially with a series of cords. The stiff-material brace (right) was the Lumbosacral Bracing System (LSO, Aspen Medical Products Inc., Irvine, CA) that consisted of plastic panels held in place by nylon straps. 
It is assumed that this brace, made of plastic panels connected by strapping, also maintains a constant brace volume during subject movement. All lumbar braces were sized and positioned on each subject following manufacturer's instructions.

\subsection{Electrodes and electrode placement}

Surface electromyographic signals were recorded from five paired sites according to previously used methodology (Cholewicki and McGill 1996): rectus abdominis (3 cm lateral to the umbilicus), external oblique (approximately $15 \mathrm{~cm}$ lateral to the umbilicus), internal oblique (approximately midway between the anterior superior iliac spine and symphysis pubis, above the inguinal ligament), thoracic erector spinae $(5 \mathrm{~cm}$ lateral to ninth thoracic spinous process) and lumbar erector spinae $(3 \mathrm{~cm}$ lateral to third lumbar spinous process). The clavicle was used as a neutral ground. All locations were shaved and cleaned with alcohol swabs prior to electrode application. sEMG signals were collected using $\mathrm{Ag} / \mathrm{AgCl}$ single-use circular electrodes (Blue Sensor M, Ambu, MD, USA) with sensor areas of $13.2 \mathrm{~mm}^{2}$ and an inter-electrode distance of approximately $1.5 \mathrm{~cm}$.

\subsection{Robot kinematics}

The parallel robot (Parallel Robotics Company, NH, USA) employed in this study allowed participants to experience global movement patterns in six degrees of freedom via unsupported sitting (Figure 2). The subjects were tested using a standardised multi-axial movement protocol which created a uniform platform ascension angle (i.e. tilt) of $15^{\circ}$ and circumduction velocity $\left(360^{\circ} / 18 \mathrm{~s}\right)$. Single preliminary trials were completed at $5^{\circ}$ and $10^{\circ}$ tilt to familiarise the subjects with the required movement patterns. Data collection began when the platform reached the $15^{\circ}$ ascension angle and continued through $360^{\circ}$ left circumduction. Left circumduction was followed by a brief pause at the initial start position. A $360^{\circ}$ right circumduction was then completed marking the end of a single trial. Circumduction speed was maintained at $20 \%$, and between each trial, subjects were given 3-min rest.

\subsection{Laser target system}

A laser pointer was strapped across the chest at a mid-sternal level of each subject. The subjects were then instructed to maintain the laser's position on a fixed target placed approximately $1 \mathrm{~m}$ in front of them. This system provided a visual cue for the subject in maintaining an upright position throughout each trial. During testing, subjects were observed and encouraged verbally to promote appropriate laser positioning.

\subsection{Safety systems}

When seated on the robotic platform (Figure 2), each participant wore an upper body climbing harness (Petzl, UT, USA) attached by safety slings (1500 kg rating) to an external support frame surrounding the robot. At all times when seated on the platform, the participant wore a certified rock-climbing helmet (C.A.M.P Technical Adventure Equipment, CO, USA).

\subsection{Surface electromyographic (sEMG) variables}

The surface electromyographic data was collected and analysed using Labview software (TX, USA) at a sampling rate of $2000 \mathrm{~Hz}$. The data were then filtered with a low-pass filter (Butterworth, 2nd order, $7 \mathrm{~Hz}$ cutoff) to better represent muscle activation dynamics (Cholewicki and McGill 1996). For each $360^{\circ}$ rotation, the primary variable was $\mathrm{sEMG}_{\text {peak }}$, which was defined as the highest intensity of muscle activity during each direction of circumduction for each trial. To be able to compare data across subjects, maximal voluntary contractions (MVC) were performed during resisted isometric contractions in six axes (flexion, extension, left/right lateral flexion and left/right rotation). The samesubject $\mathrm{sEMG}$ peak values were obtained from the un-braced and braced conditions and then expressed as percentages of the $\mathrm{sEMG}_{\text {peak }}$ values obtained during the same-subject MVCs.

\subsection{Analysis}

The average $s E M G_{\text {peak }}$ values from each set of three trials for $15^{\circ}$ of circumduction were analysed by muscle group, bracing condition and circumduction direction for each participant. A parametric analysis was performed (repeated measures analysis of variance) and significance determined for multiple and overall differences $(p<0.05)$. Specifically, paired t-tests were performed for all muscle groups, bracing conditions and movement direction. Using a Bonferoni correction with an adjustment for multiple comparisons, non-braced and braced conditions were assessed for significant differences when grouped by muscles and directions of circumduction.

\section{Results}

\subsection{Subjects}

In total, nine males and 15 females were enrolled in the study. The mean age per subject was 25.9 years (SD: \pm 1.7 ).

\subsection{Preliminary analysis}

The average peak muscle activity (\%) for all muscles in the non-braced condition was $70.72 \%$. For the soft-material brace, the average muscle activity over all muscles was 


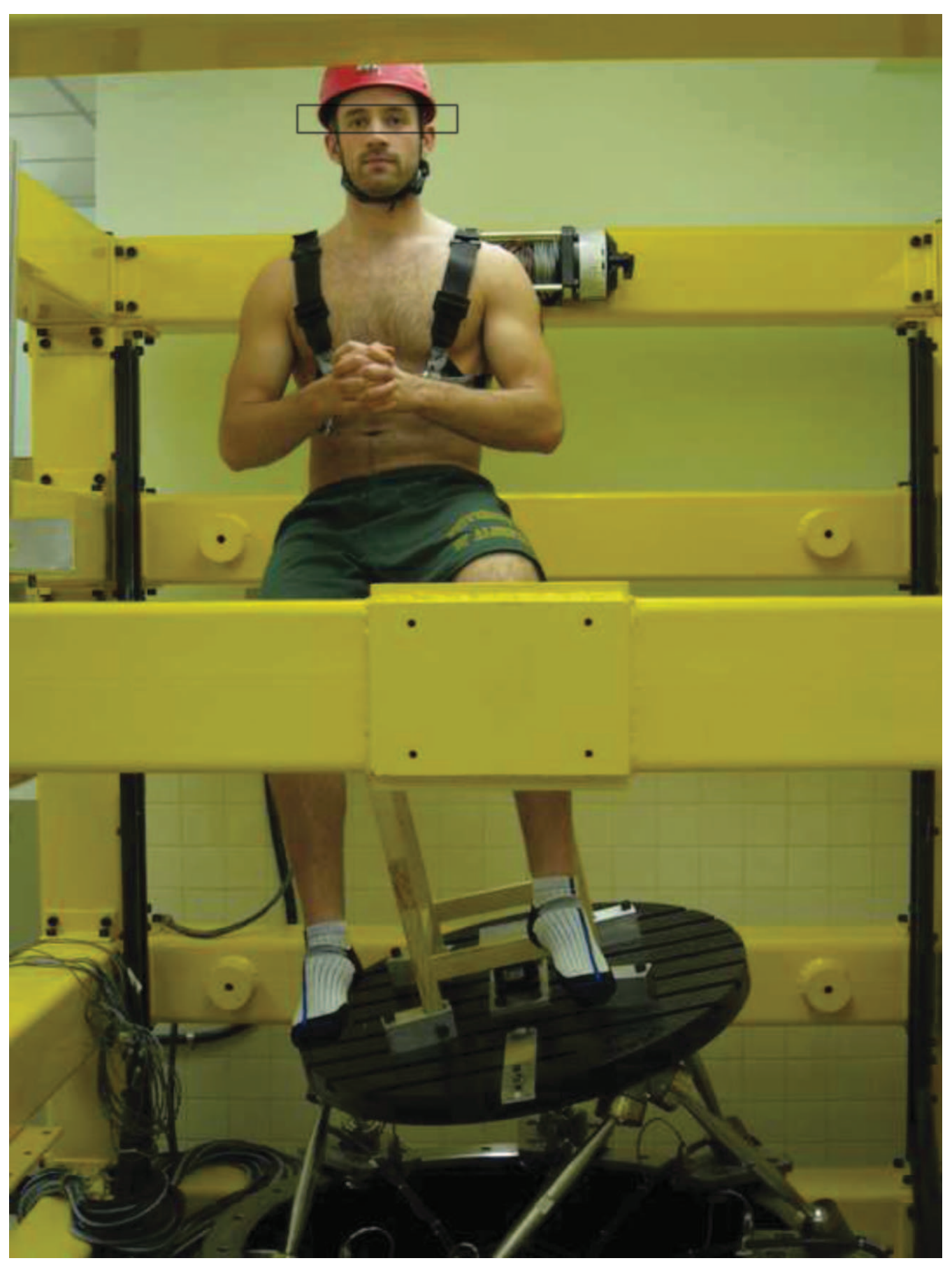

Figure 2. Subject shown in the no-brace condition while seated on the robot platform tilted to a $15^{\circ}$ ascension angle.

$51.28 \%$ for soft-material bracing and $51.39 \%$ for stiffmaterial bracing.

Averaged by muscle, the majority of muscles showed a significant reduction in muscle activity with bracing when compared with non-braced conditions (Table 2). For example, in left circumduction, the average right internal oblique activity was reduced from $65.46 \%( \pm 25.73 \%)$ to $42.54 \%$ $( \pm 33.01 \%, p<0.001)$ with soft-material bracing and to $35.35 \%( \pm 20.68 \%, p<0.001)$ with stiff-material bracing.
When not analysed by averaging, but on an individual basis, there were numerous examples where the sEMG response of a muscle was significantly higher than the baseline condition ( $>10 \%$ of baseline MVC). This paradoxical response was noted in 39 of the 240 individual muscle responses reviewed. For all muscle responses showing a decreased sEMG response (201/240, Table 3), a post-hoc analysis (Bonferoni with adjustment for multiple comparison) demonstrated a significant decrease in muscle activity 


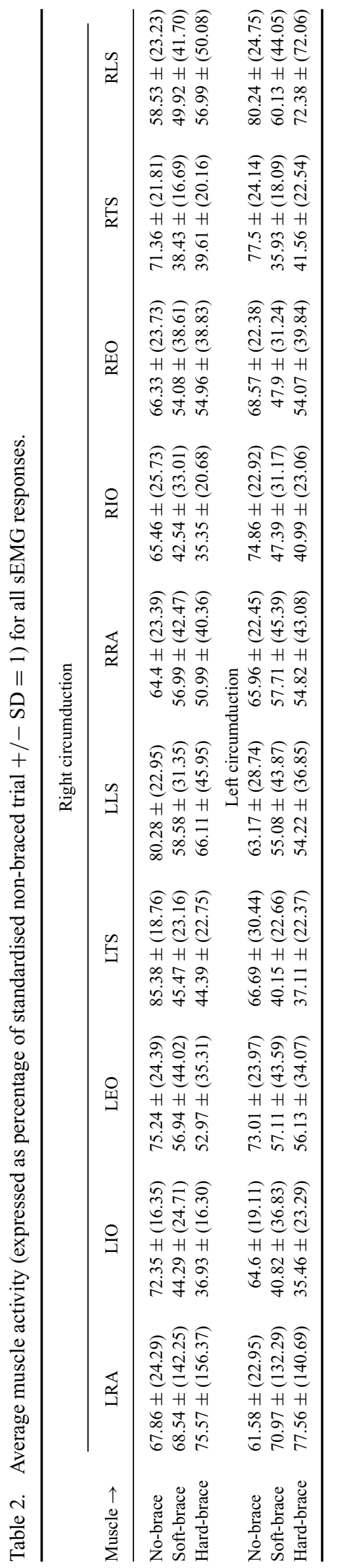




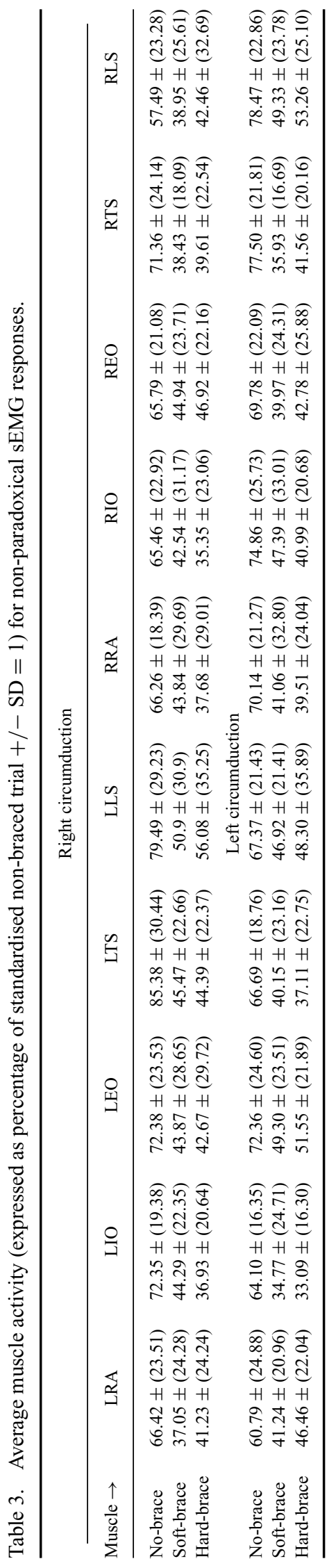


in both braced conditions in all but one muscle group (right circumduction, right rectus abdominus, $p=0.054$, Table 4). On average, muscle activity was reduced from $70.22 \%$ (non-braced) to $42.82 \%$ (soft-material brace) and $42.90 \%$ (stiff-material brace). While soft-material or stiffmaterial bracing significantly decreased muscle activity, there was no significant difference in muscle activity between the bracing conditions (given the power of the study). Muscle activity was not extinguished in either brace condition.

\section{Discussion}

\subsection{Major findings}

This study employed standardised robotic movements towards reducing variability in muscle responses observed previously with the use of lumbar bracing. For mean values, our data show that bracing decreased the sEMG response of trunk muscles as was hypothesised. In this way, our results are congruent with many other studies, but not all. Alternatively, analysis of individual muscle responses (as opposed to mean responses) demonstrated the presence of an increase in sEMG activity (paradoxical response) in a minority of muscles.

Given that paradoxical brace responses have been observed by others (Lantz and Schultz 1986), it would be doubtful that a larger sample size would have eliminated this response. More likely is the possibility that a reduction in sEMG activity is not a universal response to lumbar bracing in all subjects/muscles but can appear as such through averaging. This speculation is consistent with recent studies that suggest that asymptomatic subject pools are not homogeneous in their responses to various back pain treatments (Childs et al. 2004; Teyhen et al. 2007). Given this, the possibility exists that the traditional assumption that lumbar bracing should decrease muscle activity for all muscles in all subjects may not be accurate.

Alternatively, there are other explanations for the existence of paradoxical muscle responses. Several studies have observed sEMG activity in the trunk to both increase or decrease with the use of lumbar braces (Nachemson et al. 1983; Lantz and Schultz 1986). As these observations contradict the general assumption that bracing should result in reduced muscular activity (Lantz and Schultz 1986), several investigations have explored the possibility that bracing by itself may alter sEMG signals. In the majority of these investigations, elastic compression of trunk sEMG electrodes by itself did not influence sEMG signals (Lantz and Schultz 1986; Warren et al. 2001; Ivancic et al. 2002; Odermatt et al. 2003). Although some recent studies have elected to employ various techniques to reduce brace/electrode contact (Lavender et al. 1998; Jorgensen and Marras 2000), these efforts may not be necessary. Therefore, we conclude that brace-induced paradoxical activity is a doubtful explanation for the data observed in this study.
An additional explanation for the presence of a paradoxical response in a minority of trunk muscles is that subject's effort in maintaining an upright posture was not standardised. While it would be difficult (if not impossible) to control a subject's effort regarding this task, future studies in this area should employ technology (i.e. visual tracking) to determine if muscle activity may be related to the ability to maintain an upright posture.

At present, we do not have an explanation for the existence of a paradoxical sEMG response to lumbar bracing. Potential factors such as neuromuscular coordination, balance, strength and endurance may contribute. Given the work of others who have shown altered muscle responses to be indicative of past or impending spinal injury (Cholewicki et al. 2005), we speculate that a paradoxical response in this project may have similar implications.

In addition, the results of this study suggest that bracing reduces muscle activity, but does not extinguish it from detection using standard sEMG techniques. This finding suggests that bracing may not create a circumstance where muscles' activity would decrease to a point where muscle atrophy may occur. Given that Hides et al. (2007) demonstrated minimal reduction in spinal muscle volume after 14 days of complete bed rest, the remaining muscle activity observed in this study during the braced condition would be unlikely to result in a set of conditions that would promote atrophy. More likely, we speculate that if atrophy were to result from bracing, it may be that (1) injury is the cause of atrophy, as has been noted in multifidus (Hides et al. 1996) or (2) the brace may provide a physical cue that gives some users permission to be inactive.

\subsection{Limitations}

Although a significant difference was observed between the no-brace and the brace conditions, the study was not designed or powered to determine if the two different braces used here have equal effects. It should be noted that the constant volume condition assumed to exist with both the soft and hard braces should equally affect intra-abdominal pressure. Alternatively, different braces from different manufacturers having different stiffness values and tested under different experimental conditions may result in significantly different sEMG activity levels between brace types.

While our data suggests that bracing reduces muscle activity in most subjects, our study enrolled asymptomatic subjects that typically do not wear lumbar braces unless for a presumptive benefit related to prevention of injury. Future research with a symptomatic population using this same protocol is necessary to determine if bracing has the same effect and if paradoxical muscle responses exist in this specific population. It is unclear whether the results obtained in this study would be reproduced in a sample with low back pain, as lumbopelvic pain has been identified to affect trunk muscle activity (Hodges and Richardson 1996). 
G.N. Kawchuk et al.

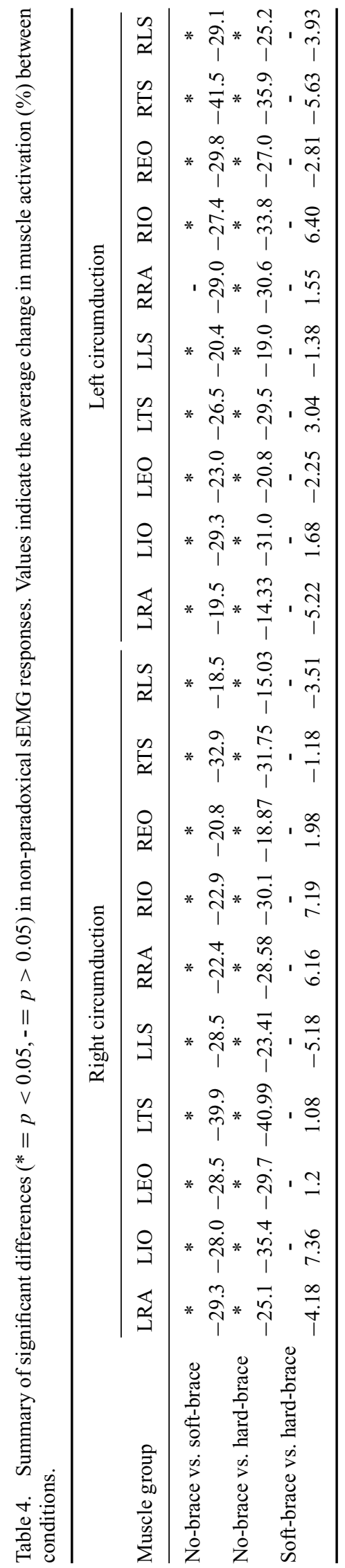


While the robot protocol itself does not replicate any common occupational or lifestyle activity, the protocol is potentially valuable, as it can query the function of all trunk muscles in a controlled environment within a short timeframe. Similarly, the value of various orthopedic, neurologic or other clinical tests may not be in replicating daily activity, but in isolating a specific anatomical feature/function (e.g. straight leg raise). Further, while the robot protocol does not elicit a maximal contraction response in most cases, the average activity level in the unbraced condition was $\sim 70 \%$ of MVC - an acceptable level of activation, given other studies which attempt to limit exertion to sub-MVC levels. In addition, by using a protocol that causes sub-maximal trunk contractions, subject fatigue is limited as is potential electrode shearing from extreme subject movements. The use of a sub-maximal protocol may allow this approach to be used with symptomatic subjects who require interventions that are optimised to reduce further injury.

\section{Conclusions}

These data suggest lumbar bracing reduces, but does not extinguish, lumbar muscle activity. The existence of a paradoxical muscle response to bracing during standardised robotic motion may partially explain inconsistencies in previous reports regarding the effect of lumbar bracing. The clinical significance of a paradoxical muscle response to lumbar bracing is unknown at this time.

\section{Acknowledgements}

We would like to thank Ms. C. Cook for her administrative support throughout the study. Also, we would like to recognise Aspen Medical Products, Inc. for supplying the braces and sEMG electrodes used in this study.

\section{References}

Anders C, Brose G, Hofmann GO, Scholle HC. 2008. Evaluation of the EMG-force relationship of trunk muscles during whole body tilt. J Biomech. 41(2):333-339.

Buchalter D, Kahanovitz N, Viola K, Dorsky S, Nordin M. 1988. Three-dimensional spinal motion measurements. Part 2: A noninvasive assessment of lumbar brace immobilization of the spine. J Spinal Disord. 1(4):284-286.

Childs JD, Fritz JM, Flynn TW, Irrgang JJ, Johnson KK, Majkowski GR, Delitto A. 2004. A clinical prediction rule to identify patients with low back pain most likely to benefit from spinal manipulation: a validation study. Ann Intern Med. 141(12):920-928.

Cholewicki J, Alvi K, Silfies SP, Bartolomei J. 2003. Comparison of motion restriction and trunk stiffness provided by three thoracolumbosacral orthoses (TLSOs). J Spinal Disord Tech. 16(5):461-468.
Cholewicki J, Silfies SP, Shah RA, Greene HS, Reeves NP, Alvi K, Goldberg B. 2005. Delayed trunk muscle reflex responses increase the risk of low back injuries. Spine 30(23):2614 2620.

Cholewicki J, McGill SM. 1996. Mechanical stability of the in vivo lumbar spine: implications for injury and chronic low back pain. Clin. Biomech. 11(1):1-15.

Hides J, Richardson C, Jull G. 1996. Multifidus muscle recovery is not automatic after resolution of acute, first-episode low back pain. Spine 21(23):2763-2769.

Hides JA, Belavý DL, Stanton W, Wilson SJ, Rittweger J, Felsenberg D, Richardson CA. 2007. Magnetic resonance imaging assessment of trunk muscles during prolonged bed rest. Spine 32(15):1687-1692.

Hodges PW, Richardson CA. 1996. Inefficient muscular stabilization of the lumbar spine associated with low back pain. A motor control evaluation of transversus abdominis. Spine 21(22):2640-2650.

Ivancic PC, Cholewicki J, Radebold A. 2002. Effects of the abdominal belt on muscle-generated spinal stability and L4/L5 joint compression force. Ergonomics 45(7): 501-513.

Jorgensen MJ, Marras WS. 2000. The effect of lumbar back support tension on trunk muscle activity. Clin Biomech. 15(4):292-294.

Kawchuk G, Clayholt L, Douglas S, Fitzgerald S, Willis A. 2008, May. Trunk muscle activity caused by 360 degree robotic tilting. Geneva: International Society for the Study of the Lumbar Spine; p. 126.

Lantz SA, Schultz AB. 1986. Lumbar spine orthosis wearing. II. Effect on trunk muscle myoelectric activity. Spine 11(8):838842.

Lavender SA, Chen SH, Li YC, Andersson GB. 1998. Trunk muscle use during pulling tasks: effects of a lifting belt and footing conditions. Hum Factors 40(1):159-172.

McGill SM, Norman RW, Sharratt MT. 1990. The effect of an abdominal belt on trunk muscle activity and intra-abdominal pressure during squat lifts. Ergonomics 33(2):147-160.

McGill S, Seguin J, Bennett G. 1994. Passive stiffness of the lumbar torso in flexion, extension, lateral bending, and axial rotation. Effect of belt wearing and breath holding. Spine 19(6):696-704.

McNair PJ, Heine PJ. 1999. Trunk proprioception: enhancement through lumbar bracing. Arch Phys Med Rehabil. 80(1):9699.

Nachemson A, Schultz A, Andersson G. 1983. Mechanical effectiveness studies of lumbar spine orthoses. Scand J Rehabil Med Suppl. 9:139-149.

Odermatt D, Mathieu PA, Beauséjour M, Labelle H, Aubin CE. 2003. Electromyography of scoliotic patients treated with a brace. J Orthop Res. 21(5):931-936.

Poppel M, de Looze M, Koes B, Smid T, Bouter L. 2000. Van Mechanism of action of lumbar supports. Spine 25(16):21032113.

Teyhen DS, Flynn TW, Childs JD, Abraham LD. 2007. Arthrokinematics in a subgroup of patients likely to benefit from a lumbar stabilization exercise program. Phys Ther. 87(3): 313-325.

Warren LP, Appling S, Oladehin A, Griffin J. 2001. Effect of soft lumbar support belt on abdominal oblique muscle activity in nonimpaired adults during squat lifting. J Orthop Sports Phys Ther. 31(6):316-323. 

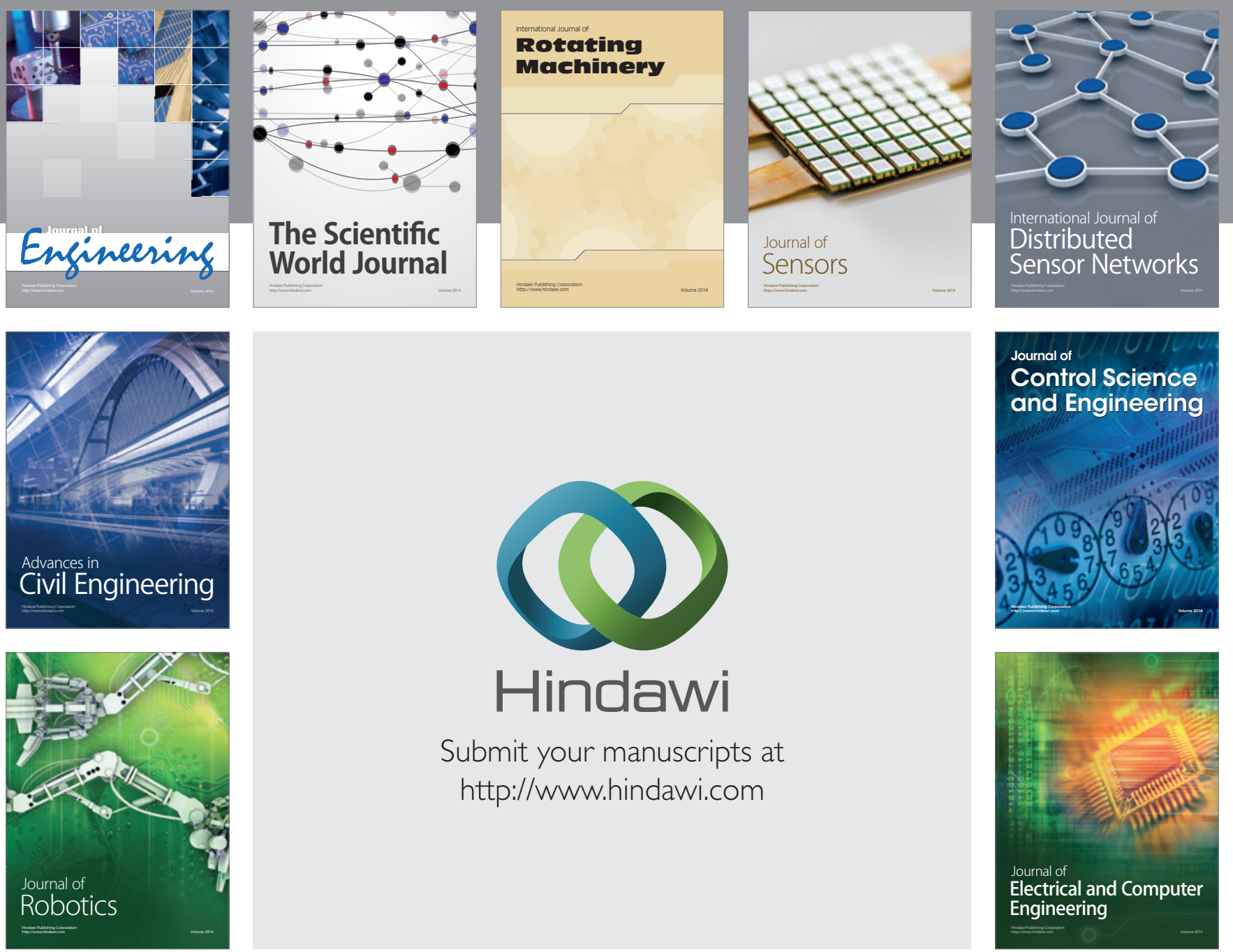

Submit your manuscripts at

http://www.hindawi.com
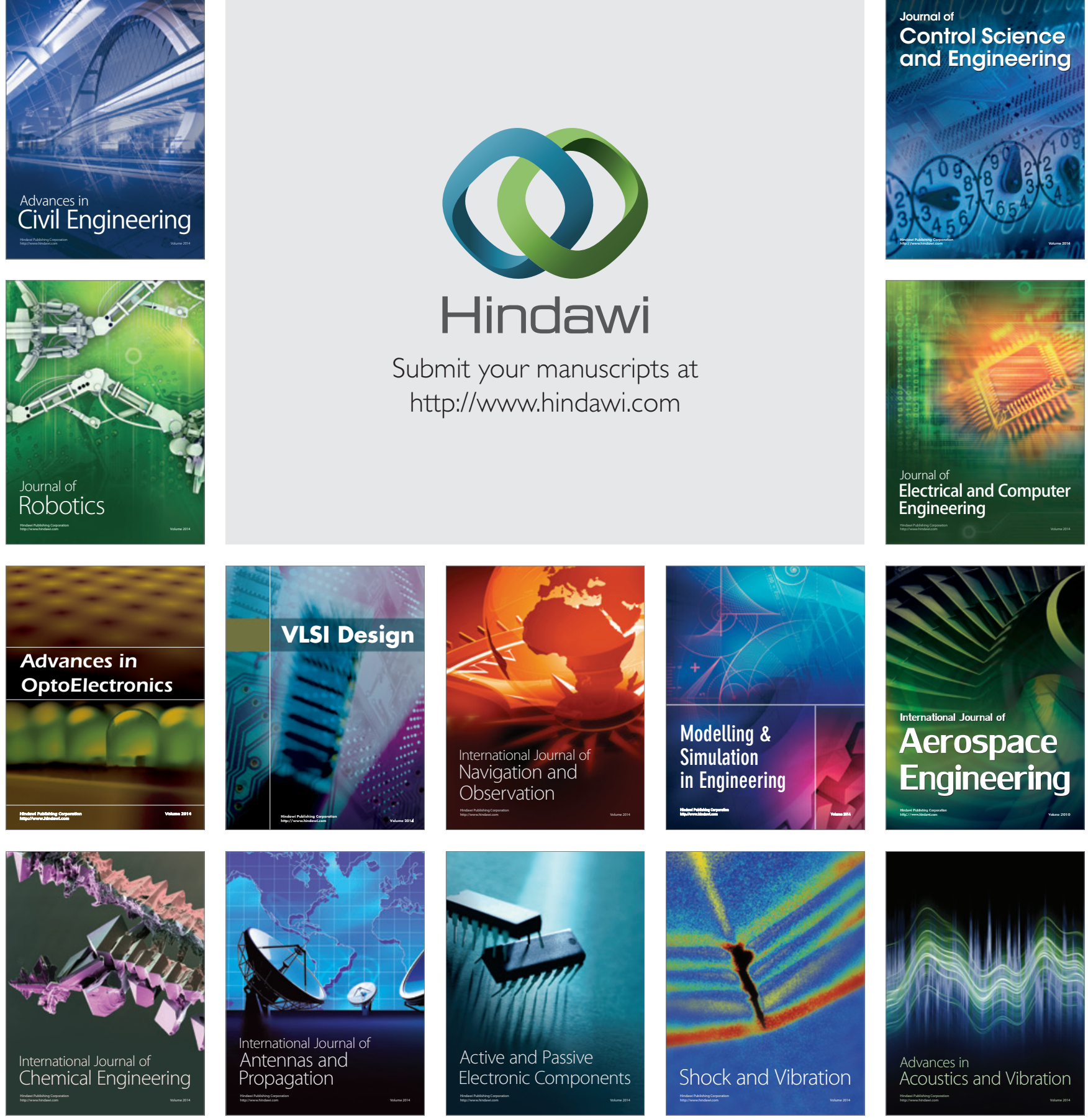